\title{
Proposta de articulação Ensino-Serviço em Enfermagem
}

\section{Articulation proposal of teaching-job in nursing}

\author{
Lucrecia Helena Loureiro $^{1}$ \\ Márcia Ribeiro Braz ${ }^{2}$ \\ Adilson da Costa Filho ${ }^{3}$ \\ Ilda Cecília Moreira da Silva ${ }^{4}$
}

Artigo

Original

Original

Paper

Palavras-chave:
Ensino em serviço
Enfermagem
Gerência
Estratégia
Saúde da Família

Resumo:

O presente estudo trata da capacitação de acadêmicos do $8^{\circ}$ período do curso de graduação em enfermagem para análise crítica e criativa, sobre os instrumentos de suporte para tomada de decisão em Saúde. Possibilitando aos futuros enfermeiros, no âmbito gerencial, atender ao planejamento das atividades desenvolvidas por aqueles que atuam na Estratégia Saúde da Família (ESF). Tem como objetivos identificar a necessidade de composição das equipes de saúde à luz de dados epidemiológicos; caracterizar a capacidade instalada das unidades de saúde da Família para atendimento ao usuário; implantar agendamento de consultas na Atenção Básica e correlacionar oferta e demanda de atendimentos especializados em Estratégia Saúde da Família. Com vistas a alcançar os objetivos propostos para este estudo, optou-se pelo estudo descritivo e exploratório, os dados foram analisados sob a luz da literatura pertinente a temática. Os resultados deste estudo apontaram as planilhas como um instrumento de fácil aplicação e eficaz na determinação de estratégias gerenciais. Contudo, os acadêmicos se mostraram sensíveis à realidade que os cerca, desejosos de mudanças consistentes, por terem consciência da necessidade de uma aprendizagem significativa, evidenciando uma atitude reflexiva, capaz de ultrapassar as dificuldades e lacunas, que diante deles se interpõem, no ensino e serviço na área da saúde pública. Conclui-se que as planilhas são um instrumento de fácil aplicação e eficazes na determinação de estratégias gerenciais. Contudo, os acadêmicos se mostraram sensíveis à realidade que os cerca, desejosos de mudanças consistentes, isto por terem consciência da necessidade de uma aprendizagem significativa, que evidencie atitude reflexiva capaz de ultrapassar as dificuldades e lacunas que diante dela se interpõem no ensino e serviço na área da saúde pública.

\section{Abstract}

This study addresses the training of students from the 8th semester undergraduate course in nursing for a critical and creative analysis about the support tools for decision in Health. Enabling future nurses, in a management level, to plan activities for the ones who work in Health Family Strategies (ESF). It aims to identify the needs for the composition of health teams, considering epidemiological data, characterize the capacity of health facilities in Health Basic Units for the customer service, install schedules of appointments in the Basic
Key words:

Teaching in Job

Management

Nursing

Health Strategy in Family

\footnotetext{
${ }^{1}$ Mestrado Profissional em Ensino em Ciências da Saúde e do Meio Ambiente - Centro Universitário de Volta Redonda/UniFOA

${ }^{2}$ Faculdade de Medicina de Petrópolis/Faculdade Arthur Sá Earp Neto - FMP/FASE

${ }^{3}$ Mestrado Profissional em Ensino em Ciências da Saúde e do Meio Ambiente - Centro Universitário de Volta Redonda/UniFOA e Departamento de Ensino de Ciências e Biologia - IBRAG/UERJ.

${ }^{4}$ Mestrado Profissional em Ensino em Ciências da Saúde e do Meio Ambiente - Centro Universitário de Volta Redonda; Faculdade de Medicina de Petrópolis/Faculdade Arthur Sá Earp Neto - FMP/FASE e Escola de Enfermagem Anna Nery/UFRJ.
}

Recebido em 09/2010

Aprovado em 08/2011

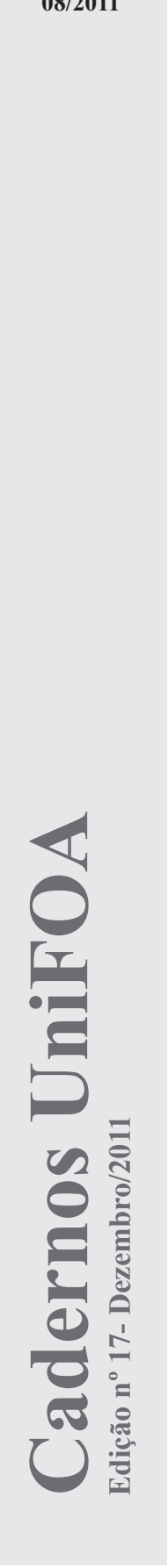


Attention and correlate supply and demand of specialized care in Health Family Strategies. In order to achieve the proposed objectives for this study a descriptive and exploratory study was performed, the data were analyzed based on a literature appropriated to the topic. The results of this study indicate the spreadsheets as instrument of easy application and efficient to determine the management strategies. However, the students were sensitive to the reality around them, eager of consistent changes, by being aware of the need for meaningful learning, presenting a reflective attitude, being able to overcome difficulties and shortcomings that come to them in the learning and working in the public health area.

\section{Introdução}

A articulação ensino-serviço, ou seja, a articulação dos processos educacional e de produção de serviço em saúde é um compromisso da Educação ou, mais particularmente, da escola com o mundo do trabalho. Seguramente essa não é uma articulação mecânica e simples. Ao contrário, enseja um grupo de questões com o qual se lida continuamente como problemática induzida. Uma delas compreende o processo educacional como aquele que deve produzir o saber pronto e acabado e o de produção de serviços de saúde como o processo do fazer (PERROTA, 2000). A integração ensino-serviço é uma estratégia para a busca dos objetivos, tanto das instituições de ensino como das instituições prestadoras de serviços de saúde, uma vez que possibilita uma formação profissional articulada à realidade social e epidemiológica do sistema local de saúde ,como também, conforme prevê a legislação do Sistema Único de Saúde (SUS), pode cooperar com o desenvolvimento da assistência de saúde uma vez que a Universidade é a instituição onde se consolidam e se constroem novos conhecimentos para transformar a realidade vivenciada.

Para cumprir essa responsabilidade, é indispensável o conhecimento das bases doutrinárias da articulação ensino-serviço, buscando compreender o encontro e desencontro entre : os elementos do processo educativo; os sujeitos que aprendem; os objetos a serem apreendidos, inclusive os conhecimentos resultantes da interação entre sujeitos e objetos; as instituições e seus atores como facilitadores e mediadores desse processo e os elementos do processo produtivo na área da saúde; os sujei- tos; os trabalhadores de saúde; os instrumentos e meios do trabalho enquanto utilização de tecnologias e saberes específicos. Certamente a compreensão desse encontro e desencontro levará ao reconhecimento desses processos (PERROTA, 2000).

A Estratégia Saúde da Família (ESF) foi implantada na cidade de Volta Redonda em 1994. Na época, as autoridades municipais sabiam muito pouco sobre o estado de saúde da sua população. Os médicos ficavam, quando muito, três horas por dia atendendo e os enfermeiros, seis horas. Ambos eram desestimulados, porque ganhavam mal. Existiam poucas unidades, o que dificultava o acesso das pessoas e acabava sobrecarregando o hospital com problemas de baixa complexidade e, muitas vezes, de simples solução. Volta Redonda tem hoje 30 unidades de saúde, sendo 09 Unidades Básicas de Saúde (UBS) e 30 unidades com ESF, compostas de 59 equipes, responsáveis pela cobertura de quase $90 \%$ da população. Nas equipes com ESF, além da formação tradicional (médico, enfermeiro, auxiliar de enfermagem e agente comunitário de saúde), também compõe o a equipe um odontólogo e um auxiliar de consultório dentário. O município, aos sete dias do mês de julho de 2008, foi subdividido em duas áreas de vigilância a saúde, denominados Distrito Sanitário Norte e Distrito Sanitário Sul.

Periodicamente, a cidade faz campanhas de prevenção de doenças e há grupos enfocando o dia a dia das famílias. Existe uma particularidade na ESF de Volta Redonda que merece destaque: o município criou, para cada unidade de saúde, a função de agente administrativo. Esse profissional cuida da área burocrática e dos problemas operacionais, um trabalho 
que antes ficava a cargo dos enfermeiros, na função gerencial. Hoje, os agentes administrativos servem de apoio à gerência.

A enfermagem brasileira tem se empenhado junto às instituições de saúde para desenvolver atividades administrativas inerentes à gerência das unidades de saúde. Não mais como um trabalho subdividido, centrado nas funções, e sim, como trabalho articulado, integrado com os demais serviços, cocompartilhado, numa relação de troca e ajuda mútua, envolvendo os diversos atores presentes neste sistema de cuidado (PROCHNOW, LEITE e ERDMANN, 2005).

Atualmente, observa-se que, no município de Volta Redonda, cerca de $100 \%$ dos gerentes são enfermeiros. Nota-se que a maioria deles é formada pelo Centro Universitário de Volta Redonda (UniFOA) e esses enfermeiros estão inseridos na Estratégia Saúde da Família (ESF). Existe um Termo de Convênio celebrado entre o município de Volta Redonda e as instituições de ensino, entre elas o Centro Universitário de Volta Redonda - UniFOA e a Secretaria Municipal de Saúde (SMS), com a inserção dos acadêmicos do curso de Enfermagem nas unidades com ESF. A partir dessa parceria, SMS e UniFOA constituiuse numa iniciativa que integra os esforços que vêm sendo desenvolvidos pelo Curso de Enfermagem na busca de melhorar a integração ensino-serviço. Para tanto, procura ampliar os espaços de difusão do trabalho desenvolvido por acadêmicos (as) e docentes das disciplinas de Saúde Coletiva I e II do Curso de Enfermagem nas Unidades Básicas de Saúde do município de Volta Redonda.

Durante a práxis docente, percebemos que não se pode formar profissionais enfermeiros, sem se discutir o campo em que vão exercer a profissão. Ensinar os futuros enfermeiros, que estão chegando ao mercado de trabalho, a gerenciar com eficiência, responsabilidade e compromisso é um desafio. Ensinar é uma tarefa árdua que exige habilidade e estudo aliado à experiência. Baseados na experiência no serviço público, bem como na gerência de serviços de saúde, acreditamos ser este estudo, uma contribuição para o crescimento profissional dos docentes e discentes inseridos no processo de ensino-serviço, assim como para a compreensão da dinâmica profissional do enfermeiro-gerente.
Algumas questões sobre o ensino prático da gerência nos levam a pensar se os acadêmicos estão preparados gerencialmente para o mercado de trabalho? Mais ainda, até onde a Academia poderá auxiliar nesta trajetória?

Diante desses questionamentos, o objetivo desta pesquisa foi verificar a possibilidade de ensinar a graduandos de enfermagem, planejar a agenda dos profissionais de saúde dos serviços de saúde nas unidades de saúde da família, por meio de planilhas, utilizando dados epidemiológicos do município de Volta Redonda. Em face da necessidade de aprimoramento e avaliação das aulas práticas de gerência em enfermagem do UniFOA, levantouse a hipótese de que essa ferramenta seja um instrumento capaz de oportunizar contribuições na referida área.

\section{Abordagem Metodológica}

Em março de 2008, devido a uma reestruturação na Secretaria Municipal de Saúde (SMS), implantou-se a área técnica, na qual os enfermeiros passaram a integrar o quadro de profissionais responsáveis por este setor dentro da SMS, com a função de adequar o atendimento na atenção básica, função esta inédita na SMS. A partir dos dados obtidos pelo censo de 2000, iniciou-se a estruturação da rede para que todos os serviços oferecidos nas unidades de saúde fossem equânimes.

Foi elaborada uma planilha adaptada do IBGE, censo 2000, da população residente por bairro, segundo a faixa etária. Essas planilhas foram preenchidas pelos agentes comunitários de saúde com dados obtidos através dos cadastramentos da população em 2008. Desta forma, acredita-se que os dados trabalhados são atualizados.

Após o preenchimento dessas planilhas, surgiu a necessidade de maior abordagem e adequação de mais informações, para uma melhor compreensão dos objetivos propostos pela área técnica. A análise dos dados foi realizada em conjunto com as gerentes das unidades, sendo assim, notamos a necessidade de adequar às agendas dos profissionais, que estavam sendo preenchidas sem os dados epidemiológicos. A partir disso, foi elaborada uma nova planilha que atendeu à gerência na

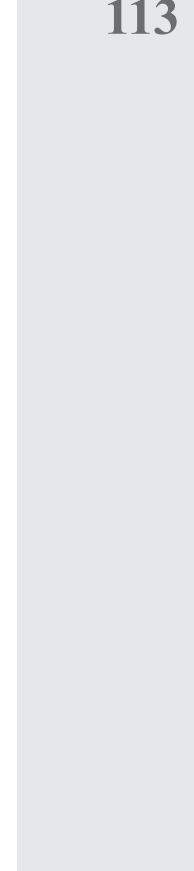

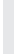 \\ ,}

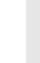
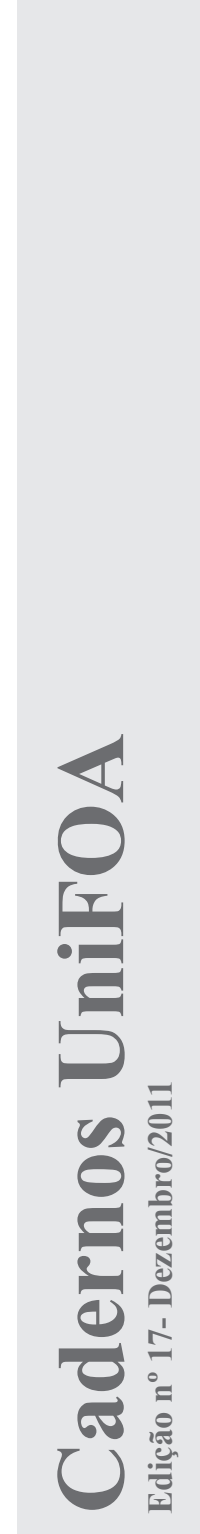
formulação das agendas dos profissionais médicos e enfermeiros, sob a luz de dados epidemiológicos, tornando-se um importante apoio para o gerenciamento, identificando assim a capacidade operacional das unidades.

Iniciou-se o monitoramento dos alunos do $8^{\circ}$ período de enfermagem, que estavam em campo de estágio na gerência, para o preenchimento dessas planilhas a fim de capacitá-los e mostrarlhes a importância dos dados que poderão ser utilizados como apoio em seu futuro profissional e como importante instrumento gerencial.

Os dados foram coletados entre agosto de 2008 a março de 2009, a partir do questionário aplicado a 30 alunos do $8^{\circ}$ período do curso de graduação em enfermagem, dos períodos diurno e noturno. Nos meses de agosto a novembro foram aplicados 24 questionários e no mês de março, 06; totalizando 30 questionários preenchidos.

Antes de iniciar a pesquisa, o projeto foi submetido à aprovação do Comitê de Ética em Pesquisa do Centro Universitário de Volta Redonda, Decreto $n^{\circ} 93.933$ de 13/01/1987, Resolução 196/96 do Conselho Nacional de Saúde (BRASIL, 1996). Os alunos que participaram foram esclarecidos sobre os objetivos da pesquisa, concordaram em participar da mesma assinando o Termo de Consentimento Livre e Esclarecido.

Foram confeccionados kits possibilitando uma melhor compreensão da pesquisa pelos acadêmicos. O kit foi composto por quatro planilhas, 01 planilha com dados e distribuição da população por sexo e faixa etária, 01 planilha para distribuição etária da população total da área de abrangência, 01 planilha para distribuição da população segundo categoria programática e 01 planilha para coleta de dados e análise da capacidade operacional das unidades. Os alunos receberam os kits e foram orientados individualmente para o preenchimento das planilhas e, ao término do mesmo, analisar a capacidade de atendimento da unidade, organização e confecção das agendas de consultas médicas e de enfermagem.

Inicialmente, os alunos analisaram suas planilhas e preencheram as demais, baseandose nos dados da população adstrita. $\mathrm{O}$ tempo utilizado para execução das atividades foi de 16 horas/aula, com 50 minutos cada. A primeira foi destinada à explicação da atividade e entrega dos kits. A segunda e terceira exclusivamente para o preenchimento das planilhas e confecção das agendas. A quarta, à resolução do questionário para avaliar as dificuldades encontradas no preenchimento das planilhas e as contribuições para o gerenciamento de serviços de saúde. Este questionário teve como objetivo avaliar a aula prática de gerência em serviços de saúde.

\section{Resultados e Discussão}

Durante a análise da concepção dos alunos acerca da contribuição das aulas práticas em gerência para o mercado de trabalho, tomou-se por parâmetro as respostas do questionário, respondido nos 04 dias de aula, no fechamento do módulo de gerência em unidade de saúde. Logo após o preenchimento das planilhas e confecção das agendas, retiramos algumas contribuições que seguem.

\footnotetext{
“... nos possibilitam vivenciar as atividades de gerência que não nos são passadas na teoria, gerando capacitação para o campo de trabalho"

(Informante 25)
}

“... ampliar o nosso conhecimento acer-
ca da disciplina, proporcionando um
maior entendimento do que é gerência
de enfermagem e como utilizá-la no dia
a dia”.
(Informante 24)
"... contribui para facilitar a visão de
como se deve gerenciar uma unidade
da forma mais correta".
(Informante 7)

Portanto, a gerência como instrumento do processo de trabalho na organização de serviços de saúde, implica na tomada de decisões que afetam a estrutura, o processo de produção e o produto de um sistema, visando ações que possibilitem intervenções impactantes no processo de trabalho em saúde, ou seja, viabilizar meios para prestação da assistência à clientela com eficiência, eficácia e efetividade, a fim de possibilitar a satisfação das necessidades de saúde desta clientela (ERDMANN, 2001). 
Nessa mesma linha de pensamento, Ceccim e Feuerwerker (2004) comentam que aprender a aprender o SUS exige e oportuniza ao graduando de Enfermagem questionar indicadores de saúde abordados na epidemiologia, em diferentes realidades- como na unidade de internação, no preenchimento burocrático de um procedimento, na necessidade de realizar visita domiciliar à família, entre outras situações- que tem sentido, além do aspecto individual de cuidado, e que ao ser executado, nem sempre é pontuado na perspectiva coletiva.

Para Spagnol (apud HECK et al., 2009) o comprometimento do Enfermeiro com a proposição do SUS é uma necessidade eminente, que vem sendo repetidamente discutida. Apesar das discussões já existentes acerca desta temática, ainda são desafios contemporâneos da gestão: superar o modelo tradicional centrado no financeiro e de administração de material, desenvolver linhas diferenciadas com enfoque nas especificidades, superar o reducionismo na atenção a saúde e criar práticas democráticas na dinâmica gerencial (ABRAHÃO et al., 2008). Feldman, Ruthes e Cunha (2008) corroboram este pensamento afirmando que o enfermeiro supera estes desafios da gestão quando aplica a criatividade e inovação nos serviços de saúde.

Ao serem questionados sobre quais dificuldades foram encontradas no preenchimento das planilhas, com os dados epidemiológicos para a implementação da agenda dos profissionais da ESF, 62\% dos alunos responderam que a dificuldade apresentada estava na falta de prática e de conhecimento especifico; $23 \%$ responderam que após a explicação do objetivo da planilha, não houve dificuldade no preenchimento e elas são de fácil entendimento; $15 \%$ responderam a pergunta com resposta incoerente, Conclui-se que os alunos não entenderam o que foi proposto.

A análise da última pergunta do questionário: "Quais as contribuições das planilhas para o gerenciamento de serviços de saúde?", revelou que todos os alunos responderam que as planilhas simplificam e agilizam o serviço e descreveram:

“... que as planilhas ajudam na interpretação dos dados, dando a visão do todo, agilizando o atendimento dos usuários” (Informante 22)
“... são ferramentas essenciais para que todos consigam ser atendidos, assim é possível fazer um bom gerenciamento" (Informante 24)

“... simplificam a interpretação dos dados epidemiológicos"

(Informante 1)

Consequentemente, a programação das agendas constitui-se num instrumento que orienta a condução do processo de organização dos serviços, na medida em que estabelece os objetivos a serem alcançados, bem como as estratégias a serem utilizadas. Igualmente, todos os alunos que participaram do estudo revelam entender a extrema importância em desenvolver a programação da agenda dos profissionais que atuam no ESF. Os recursos que as planilhas proporcionam são importantes para o gerente do ESF, a fim de conhecer a capacidade da sua unidade para o desenvolvimento planejado das atividades, através de dados epidemiológicos, tendo condições para direcionar as atividades.

A epidemiologia se destaca pelo seu emprego e/ou utilização para planejar, implementar e avaliar o cuidado à saúde, a indivíduos e a populações; e pelo seu significativo papel na complementaridade de áreas de conhecimento. Considerando o aspecto global do trabalho do enfermeiro, como suas ações na vigilância epidemiológica, na administração de serviços e na assistência de enfermagem, a epidemiologia tem um importante papel nesses processos, não somente nos aspectos de prevenção e vigilância das enfermidades ou ocorrências, mas também na avaliação e análise do impacto de suas ações. É aplicada para identificar necessidades, riscos, definir prioridades em saúde da população, melhorar a utilização dos recursos disponíveis, entre outros (SOUZA et al., 2008).

Torna-se imprescindível ao aluno conhecer e aprender o trabalho da gerência em saúde, uma vez que, em breve, cabe a ele assumir a gerência da unidade e a organização dessa assistência. Assim, o processo de agendamento do trabalho dos profissionais em saúde integraliza a ação e complementa o processo de produção. A organização e a divisão do processo de trabalho definem-se pelo objetivo final que se quer atingir. 
Estudos de Kloetzel et al., Matsuda et al. e Mendes et al. (apud VOLPATO et al., 2010) corroboram com os resultados desta pesquisa e mencionam a importância da pontualidade no atendimento. Os achados também estão de acordo com as pesquisas de Tengan (2008) e Schwartz et al. (2010), que identificaram a facilidade para agendar consulta de tratamento especializado e no serviço como aspectos de grande importância ao usuário. Já na década de 90, Franco e Campos (1998), em seus estudos, mostraram que a demora no atendimento, excesso de pessoas para serem atendidas e dificuldade para conseguir consulta em serviço especializado são os maiores problemas enfrentados pelos usuários.

A gerência é uma das atividades que fazem parte da rotina do enfermeiro. Neste sentido, a importância desta refere-se à ação que torna viável e factível o melhor uso dos recursos para atingir os objetivos planejados e propostos. Para uma nova concepção de gerência, é preciso aceitar que a função administrativa se constitua em elemento essencial para o alcance dos objetivos e que o planejamento correto das agendas proporciona ao gerente estabelecer a capacidade operacional da unidade à luz de dados epidemiológicos.

\section{Considerações Finais}

As planilhas, utilizadas como ferramenta didático-pedagógicas para fixação e revisão de conteúdos de gerência, produzem resultados significativamente eficazes, contribuindo para a apropriação de conhecimentos dos acadêmicos na gerência das unidades da ESF.

Entende-se que a organização geral das atividades, "aulas práticas", específicas do estágio em gerência, possibilitaram a análise da ação educativa e a elaboração de sínteses pelos alunos.

As aulas práticas de gerência foram atividades consideradas essenciais, pois possibilitaram aos alunos debates coletivos, enriquecidos por diferentes interpretações e análises. No entanto, o desenvolvimento dessa atividade exigiu articulação constante entre o professor envolvido e os acadêmicos, o que demandou tempo, muitas vezes não disponibilizado pelo centro universitário.
As aulas práticas de gerência foram percebidas por todos os graduandos como um momento privilegiado, pois ele alimentava as demais atividades e possibilitava aos alunos o contato com situações reais e a análise desta. Verificamos a relevância da experiência prática dos graduandos como fonte de aprendizagem, possibilitado constatações e reflexões sobre a realidade.

Este estudo pode ser considerado um instrumento de auxílio para a reflexão e mudanças das aulas práticas de gerência, atendendo a demanda de formar profissionais com um perfil diferenciado, com vista na qualidade da assistência. Um aspecto relevante é a ênfase que deve ser dada à criação de formas e metodologias de processos pedagógicos, visando à transformação de práticas no cotidiano, baseadas na mudança de paradigmas dos alunos que irão trabalhar em saúde. Nesse sentido, a educação in loco apresenta-se como instrumento valioso para se chegar a esta mudança de conduta. Os resultados desta pesquisa frisam a necessidade de se mudar a prática das aulas de gerência em unidades de saúde, sobretudo se tratando dos alunos que vão atuar no Programa Estratégia Saúde da Família.

Entende-se, pois, que as planilhas podem ser utilizadas pelos profissionais de saúde no seu processo de trabalho, no sentido de identificar e evidenciar as demandas de consultas programadas para cada profissional, auxiliando no diagnóstico da realidade em todas as dimensões, contudo, é importante destacar que a planilha é um elemento complementar no processo de trabalho da gerência e não dispensa outros recursos tecnológicos, como por exemplo, o computador. Assim o instrumento deve ser utilizado para ampliar o trabalho gerencial.

Dessa forma, o instrumento proposto revela-se como uma ferramenta auxiliar para o trabalho do gerente, não interferindo na autonomia do enfermeiro; uma vez que não define previamente a totalidade do processo de trabalho, mas pode ser um instrumento importante para o diagnóstico e o planejamento das ações gerenciais.

Por fim, cabe destacar, os alunos indicaram as planilhas como um instrumento de fácil aplicação e eficaz na determinação de estratégias gerenciais e se mostraram sensíveis à realidade que os cerca, desejosos de mudanças consistentes, isto por terem consciência da necessidade 
de uma aprendizagem significativa, que evidencie atitude reflexiva capaz de ultrapassar as dificuldades e lacunas que diante dela se interpõem no ensino e serviço na área da saúde pública.

O presente estudo com graduandos de enfermagem nas unidades de Estratégia Saúde

\section{Referências}

1. ABRAHÃO, A.L; MARTINS, C.M; GEISLER, A. O sujeito na literatura acadêmica sobre gestão em saúde: notas para a questão da autonomia. Ciênc. saúde coletiva. 2008;13(1)247-57.

2. BRASIL. Ministério da Saúde. Conselho Nacional de Saúde. Normas de pesquisa envolvendo seres humanos. Res. CNS 196/96. Bioética 1996; 4 Suppl:15-25.

3. CECCIM R, B; FEUERWERKER, L.C.M. Mudanças na graduação das profissões sob o eixo da integralidade.

Cad. Saúde Pública. 2004;20(5):140010.

4. ERDMANN, A. L. Administração do processo de trabalho e da assistência de enfermagem.In: Santos I, organizadora. Enfermagem fundamental. São Paulo: Atheneu; 2001. p. 143-55.

5. FELDMAN, L.B; RUTHES, R.M; CUNHA, I.C.K.O. Criatividade e inovação: competências na gestão de enfermagem. Rev Bras Enferm. 2008;61(2):239-42.

6. FRANCO, S.C; CAMPOS, G.W. Avaliação da qualidade de atendimento ambulatorial em pediatria em um hospital universitário. Cad Saúde Pública 1998; 14:61-70.

7. HECK, R.M; JARDIM, V.R; DILÉLIO, A.S; SILVA, S.J. Uso de metodologia ativa na disciplina gerenciamento de enfermagem em saúde coletiva da FEO/UFPEL. Rev. Eletr. Enf. [Internet]. 2009;11(2):429-34. Available from: http://www.fen.ufg.br/ revista/v11/n2/v11n2a27.htm da Família confirma a hipótese inicial de que as planilhas utilizando dados epidemiológicos é um instrumento capaz de oportunizar contribuições na referida área, além de auxiliar nas estratégias que devem ser adotadas para a melhoria da instituição.

8. PERROTA, M.P. Novos Fundamentos para uma didática crítica. Rio de Janeiro: 7 Letras, 2000.

9. PROCHNOW,A.G;LEITE, J.L;ERDMANN, A.L. Teoria interpretativa de Geertze a gerência do cuidado: visualizando a prática social do enfermeiro. Rev latino-am enfermagem 2005 julho-agosto; 13(4):583-90.

10. SOUZA, S.S et al. A epidemiologia como instrumental na produção de conhecimento em enfermagem. R Enferm UERJ, Rio de Janeiro, 2008 jan/mar; 16(1):58-63.

11. SCHWARTZ, T.D et al . Estratégia Saúde da Família: avaliando o acesso ao SUS a partir da percepção dos usuários da Unidade de Saúde de Resistência, na região de São Pedro, no município de Vitória (ES). Ciênc. saúde coletiva, Rio de Janeiro, v. 15, n. 4, July 2010. Available from $<$ http://www. scielo.br/scielo. Access on 31 May 2011.

12. TENGAN, C. Abordagem teórica e aplicação de um método de qualidade em serviço público odontológico [Tese de Doutorado]. Piracicaba: Faculdade de Odontologia de Piracicaba, Universidade Estadual de Campinas; 2008.

13. VOLPATO, L.F et al . Planejamento da qualidade nas unidades de saúde da família, utilizando o Desdobramento da Função Qualidade (QFD). Cad. Saúde Pública, Rio de Janeiro, v. 26, n. 8, Aug. 2010. Available from <http://www.scielosp.org/ scielo. Access on 31 May 2011.

\section{Endereço para Correspondência:}

Lucrécia Helena Loureiro

lucreciahelena@hotmail.com

Campus Universitário Olezio Galotti

Av. Paulo Erlei Alves Abrantes, $n^{\circ} 1325$

Três Poços - Volta Redonda - RJ

CEP: 27240-000 